\title{
A Pulsed Spectrometer Designed for Feedback NQR
}

\author{
J. L. Schiano and M. D. Ginsberg ${ }^{a}$ \\ Department of Electrical Engineering, The Pennsylvania State University, \\ 227D Electrical Engineering West, University Park, PA 16802, USA \\ ${ }^{a}$ United States Army Construction Engineering Research Laboratories, \\ PO Box 9005, Champaign, IL 61826, USA
}

Reprint requests to Prof. J. L. S.; Fax: (814) 865-7065; E-mail: schiano@ steinmetz.ee.psu.edu

Z. Naturforsch. 55 a, 61-66 (2000); received August 28, 1999

Presented at the XVth International Symposium on Nuclear Quadrupole Interactions, Leipzig, Germany, July 25 - 30, 1999.

\begin{abstract}
A pulsed NQR spectrometer specifically designed to facilitate real-time tuning of pulse sequence parameters is described. A modular approach based on the interconnection of several rack-mounted blocks provides easy access to all spectrometer signals and simplifies the task of modifying the spectrometer design. We also present experimental data that demonstrates the ability of the spectrometer to increase the signal to noise ratio of $\mathrm{NQR}$ measurements by automatically adjusting the pulse width in the strong off-resonant comb pulse sequence.
\end{abstract}

Key words: Nuclear Quadrupole Resonance; Spectrometer Design; Real-time Programmable Pulse Sequence Generator

\section{Introduction}

An NQR spectrometer that can automatically adjust pulse parameters in response to the observed magnetization signals is described. Feedback control of pulse parameters is advantageous when the pulse parameters that maximize the signal-to-noise ratio (SNR) of the NQR measurements cannot be determined before the pulse sequence is applied. For example, NQR provides a means for detecting minimalmetal landmines. In this application, the pulse parameters that yield the largest SNR depend on unknown factors, such as the temperature and burial depth of the mine [1].

Commercial spectrometers are typically not designed to facilitate real-time control of pulse sequence parameters. In addition, during the last forty years NMR and NQR spectrometers have evolved from several pieces of interconnected instrumentation to a single enclosed box appended to a computer workstation. As a result, it is difficult to both monitor and manipulate spectrometer signals in a closed-loop system. We present a spectrometer design that overcomes these limitations by providing the flexibility to monitor and manipulate signals during the pulse sequence.

The most notable characteristic of the spectrometer is its ability to change pulse sequence parameters while the experiment is in progress. A real-time programmable pulse sequence generator (RPSG) orchestrates the various activities that occur during the pulse sequence and enables real-time adjustments of pulse parameters. The spectrometer consists of several interconnected rack-mounted building blocks such as broadband mixers and RF gates. Interconnections between modules are made with $50 \Omega$ BNC-to-BNC jumper cables. The modular design allows rapid prototyping of different system configurations and provides complete access to all signals within the spectrometer.

The spectrometer operates in a frequency range from $2 \mathrm{MHz}$ to $200 \mathrm{MHz}$ and can produce a 50 Gauss field in a cylindrical sample 1 inch in diameter and 3.25 inches long. Although designed to develop feedback control algorithms that optimize the performance of nitrogen-14 NQR pulse sequences, the spectrometer has also been used to search for $\mathrm{NQR}$ transitions. The real-time programmability that 


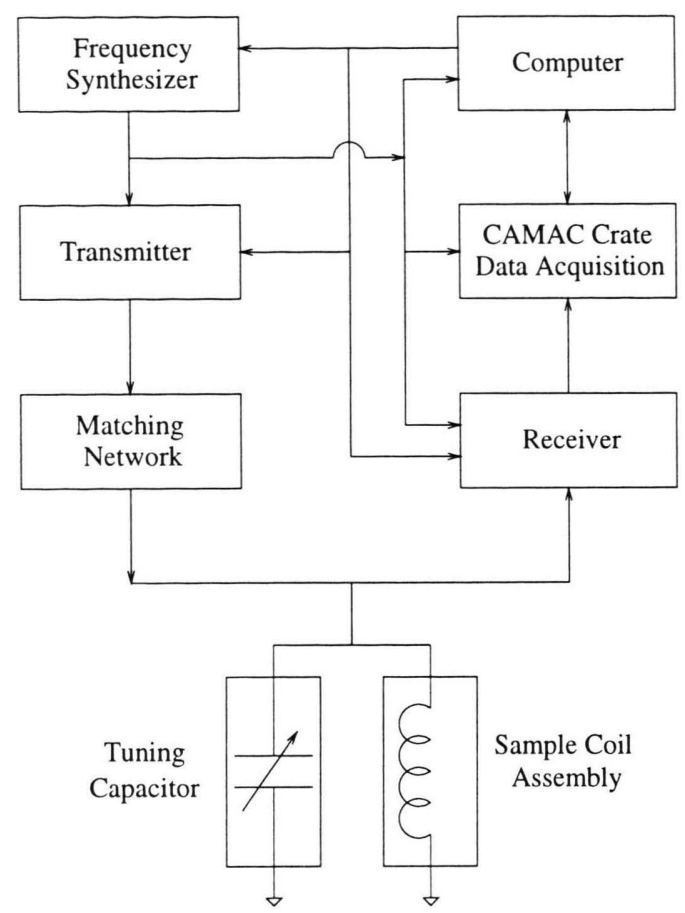

Fig. 1. Simplified block diagram of the spectrometer.

enables the implementation of feedback control algorithms also provides the capability of automating the search for NQR transitions.

\section{System Architecture}

The testbed spectrometer is a modification of the spectrometers described by Petersen [2], Harding [3], and Hirschfeld [4]. Figure 1 shows a simplified block diagram of the NQR spectrometer. Both the transmitter and receiver are single-conversion heterodyne systems. A matching network transforms the $50 \Omega$ output impedance of the transmitter to the high impedance load presented by the parallel combination of the sample coil inductor and tuning capacitor. Nuclear magnetization signals induced in the sample coil are coupled to the high impedance in a t of the receiver. The receiver contains a phase-sensitive detector that allows the acquisition of both the in-phase and quadrature components of the magnetization signal. A dedicated computer-aided measurement and control (CAMAC) crate is used to digitize and average both components of the magnetization signal. The digitized signals are transferred to the computer system using direct memory access (DMA) control to maximize data throughput. A dedicated digital signal processing unit con-

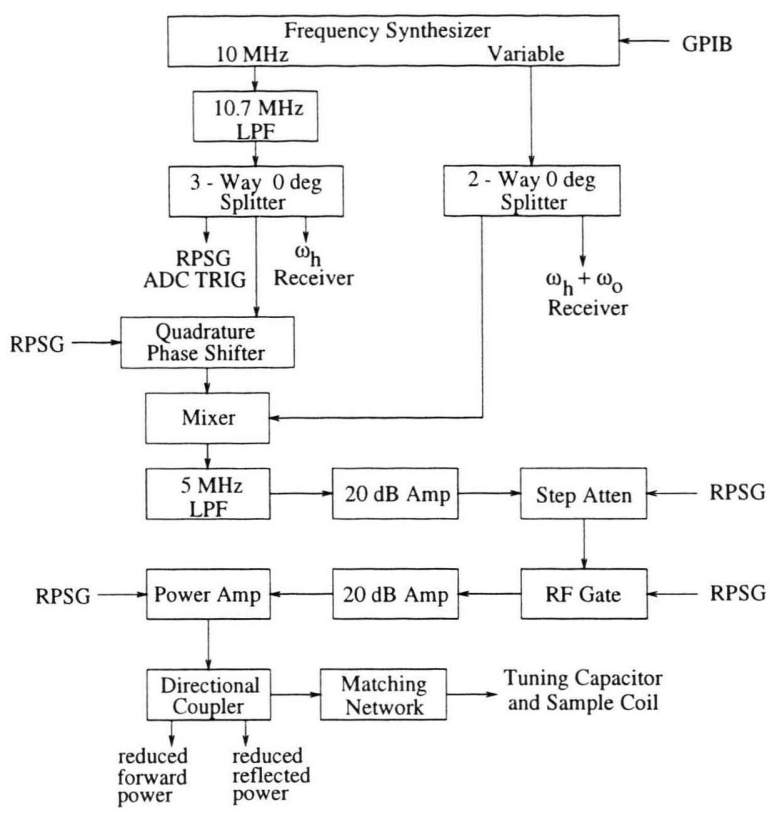

Fig. 2. Block diagram of the single-conversion heterodyne transmitter.

tained within the computer system enables real-time frequency analysis of the acquired data. Operation of the spectrometer is coordinated by the RPSG, which is also located in the computer and phase-locked to the local oscillator signal used by the receiver and transmitter.

A detailed block diagram of the transmitter is shown in Fig. 2, where each block corresponds to a separate rack-mounted module. A single-conversion heterodyne design is used for two reasons. First, it is easier to introduce phase shifts into the RF signal at a fixed intermediate frequency (IF), rather than designing a phase shifter that operates over the entire bandwidth of the spectrometer. Second, the heterodyne system prevents coherent interference signals from being produced in the receiver during detection of the NQR signal.

A frequency synthesizer (Programmed Test Sources model PTS-250) provides a $10 \mathrm{MHz}$ master oscillator signal as well as a variable frequency output that spans from $1 \mathrm{MHz}$ to $250 \mathrm{MHz}$. The $10 \mathrm{MHz}$ signal provides a master clock signal for both the RPSG and the ADC trigger generator. The $10 \mathrm{MHz}$ signal also serves as the local oscillator frequency used in the receiver and transmitter sections of the spectrometer. The variable frequency output of the frequency synthesizer is set to the sum of the heterodyne (local 


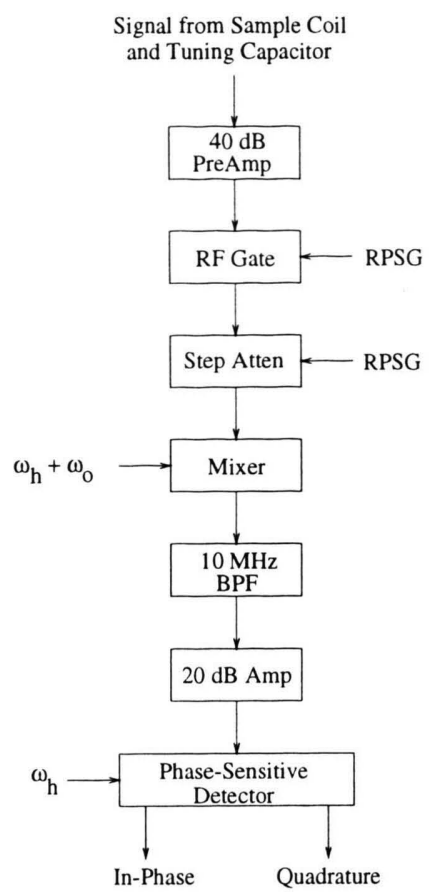

Fig. 3. Components of the single-conversion heterodyne receiver with a quadrature phase-sensitive detector.

oscillator) frequency $\omega_{\mathrm{h}}$ and the operating frequency $\omega_{\mathrm{o}}$. Both the amplitude and frequency of the variable output are controlled by the computer through a GPIB (General Purpose Interface Bus) based on the IEEE-488 standard.

The output of the quadrature phase shifter is mixed with the variable frequency output of the synthesizer and low-pass filtered to produce a signal at the operating frequency $\omega_{0}$. The mixer is an active device that uses an Analog Devices AD834 linear multiplier, which has an operating frequency range of DC to $500 \mathrm{MHz}$. Two $20 \mathrm{~dB}$ low noise amplifiers (MiniCircuits ZFL-500) and a programmable attenuator (JFW Industries 50P-1130) set the power level of the RF pulses. The RF signal is gated using a RF switch (Watkins-Johnson S1) which has an isolation of $90 \mathrm{~dB}$ and a switching speed less than 100 ns.

The RF pulse is passed to a gated $1 \mathrm{~kW}$ pulsed amplifier (Amplifier Research 1000LP). The power amplifier is connected to the matching network through a directional coupler (Amplifier Research DC2500). The coupler provides measurements of forward and reflected power that are used to tune the matching network. The matching network serves two functions [5]. First, it matches the $50 \Omega$ output impedance of the power amplifier to the impedance of the tuned sample coil assembly. Second, it steps-up the output voltage of the power amplifier by a factor of ten to increase the magnetic field produced by the sample coil.

Figure 3 shows the basic components of the receiver. The sample coil is capacitively coupled to a high-impedance input amplifier with a gain of $40 \mathrm{~dB}$. During the transmission of an RF pulse, the receiver is protected by a crossed-diode pair. The output of the preamplifier is connected to an RF gate that is only switched on during reception of NQR signals in order to avoid saturating other receiver modules during RF pulses.

The output of the receiver RF gate is connected to a constant phase step-attenuator that controls the gain of the receiver. The output of the attenuator is mixed with the variable frequency output of the synthesizer to produce an intermediate frequency (IF) signal that is centered at $\omega_{\mathrm{h}}=10 \mathrm{MHz}$. The $10.7 \mathrm{MHz}$ bandpass filter (Mini-Circuits BBP-10.7) eliminates the high frequency component centered at $2 \omega_{0}+\omega+h$.

The filtered IF signal is amplified by $20 \mathrm{~dB}$ (MiniCircuits ZFL-500) and passed to a quadrature phase detection circuit that uses a $2-W a y-90^{\circ}$ splitter (MiniCircuits PSCQ-2-10.5) and two analog multipliers (Analog Devices AD835). The in-phase and quadrature output signals are DC coupled so that they are not distorted when operating exactly on resonance. In addition to the baseband NQR signal, each output of the detector also has a component centered at $20 \mathrm{MHz}$ which is eliminated by a low-pass filter in the data acquisition system.

The in-phase and quadrature components of the receiver output are passed to the data acquisition system shown in Figure 4. Each module shown in this diagram is mounted in a 25-slot computer-aided measurement and control (CAMAC) crate (LeCroy 8025). The receiver output signals enter the data acquisition system through a two channel programmable amplifier with antialiasing filters (DSP Technology $1402 \mathrm{E}$ ) which is programmed by the system computer through the CAMAC controller module. The module uses pre-filter and post-filter amplifiers to achieve high sensitivity without saturation. The gain of both amplifiers can be controlled by the host computer. In addition, the amplifier can also be programmed to add a DC offset to the input signal. This feature is used to null the DC offset at the phase-sensitive detector outputs. The state-variable filter contained in the module 


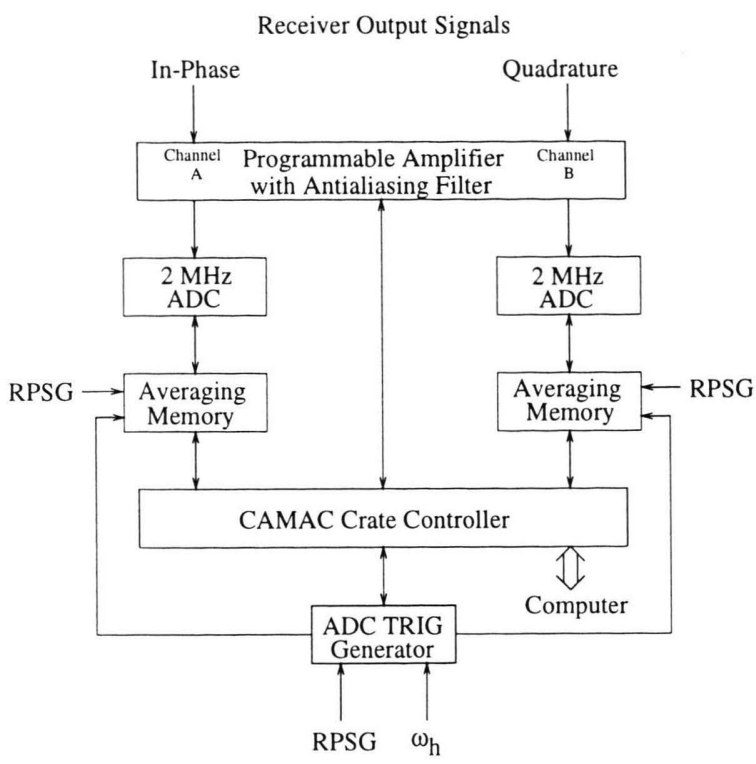

Fig. 4. The data acquisition system is contained within a single CAMAC crate.

can implement either a Butterworth or Bessel lowpass filter. The number of poles and cut-off frequency of the filter can be programmed.

The filtered and amplified signals are passed to separate analog-to-digital converters (ADCs) that each have a maximum sample rate of $2 \mathrm{MHz}$ (DSP Technology 2824). The ADC trigger generator controls the number of data points acquired and the sample rate. The signal averaging modules (DSP Technology 4101) can be programmed to add or subtract acquired waveforms coherently, and each module contains a 24 bit, $32 \mathrm{k}$ sample memory.

The Pentium-based computer system that controls the operation of the spectrometer is shown in Figure 5. The computer is equipped with $40 \mathrm{MB}$ of RAM and a 2 GB hard drive. In order to accommodate a large number of peripheral devices, a separate AT Expansion Chassis is connected to the Pentium computer system.

The AT expansion chassis contains the GPIB interface which allows the computer to set both the amplitude and frequency of the synthesizer. The DSP board enables the system to perform real-time frequency analysis of the signal data transferred from the CAMAC crate. The expansion chassis also houses the RPSG which is an integral part of the spectrometer and serves to orchestrate the various activities

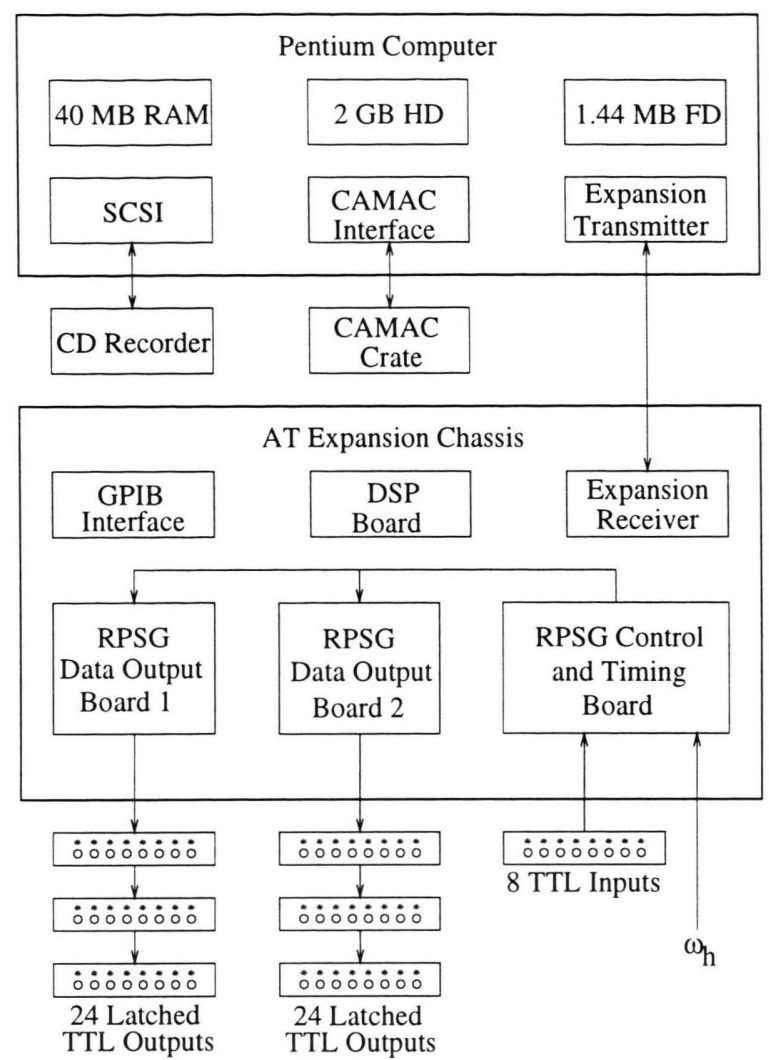

Fig. 5. The Pentium based computer system contains several specialized cards including the RPSG system and DSP processor.

that occur during the pulse sequence. In comparison to the standard pulse sequence generator, the innovative feature of the RPSG is that pulse parameters can be altered by the computer program during the pulse sequence. This is accomplished by overlapping the memory of the RPSG, where pulse sequence parameters are stored, with the memory of the host computer.

The RPSG consists of a control and timing board and up to seven data boards. Each data board is connected to the control and timing board on a local bus and provides 24 latched TTL-level outputs. The RPSG can generate up to 8192 events in a single pulse sequence, where each event is defined by the state of the latched TTL-level output lines and the duration of that state. The control and timing board accepts eight TTL-level input lines which can be used to monitor the latched TTL-level output lines as well as generate hardware interrupt requests to the host computer. The use of interrupts allows the computer to work on other 


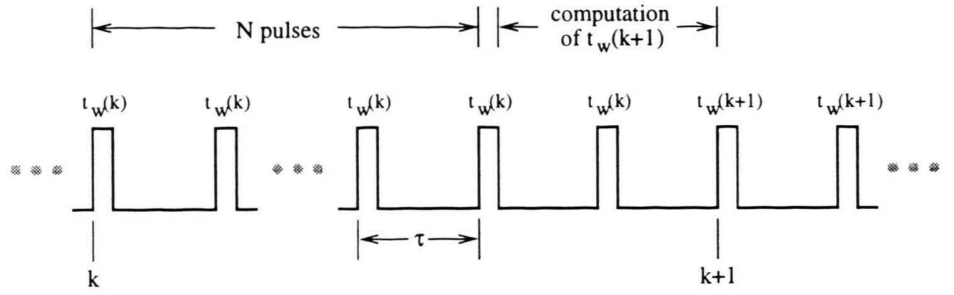

Fig. 6. SORC pulse sequence used in the control experiments. tasks while the RPSG is controlling the experiment. The operation of the RPSG is controlled through software using a control and status register (CSR). The CSR is used to direct the storage of data in the RPSG memory, to start and stop the pulse sequence, and to enable interrupt operation. By reading the CSR, the state of the eight TTL-level input lines to the RPSG can be determined. Although the RPSG is intended for use with an IBM personal computer or compatible system, the design strategy can be exported to other computer platforms.

The performance specifications of the RPSG are similar to those of a typical PSG. When terminated into a $50 \Omega$ load, the rise (fall) time of the TTL pulse is less than 10 nanoseconds. The accuracy and repeatability of the event duration is determined by an external clock. Using the $10 \mathrm{MHz}$ master clock, the minimum event time is 0.2 microseconds while the maximum event time is approximately 215 seconds. In contrast to spectrometers using a standard PSG, the RPSG allows the host computer to modify the state or duration of any event up to $30 \mu$ s before that event occurs.

\section{Experimental Results}

The testbed spectrometer is a versatile system that can implement an arbitrary pulse sequence such as the spin-locked spin echo (SLSE) [6] or strong offresonant comb (SORC) [7] pulse sequences. The most significant feature of the spectrometer is the ability to alter pulse parameters in response to the measured magnetization. This feature has enabled the use of feedback control algorithms that automatically adjust pulse parameters to maximize the signal-to-noise ratio of $\mathrm{NQR}$ measurements using the SORC sequence [8 - 10].

As an example, the pulse width in the SORC sequence shown in Fig. 6 was automatically adjusted using measurements of the SORC signal. The pulse

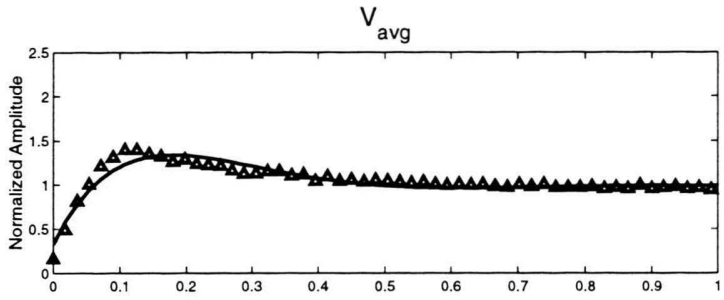

Tuning History

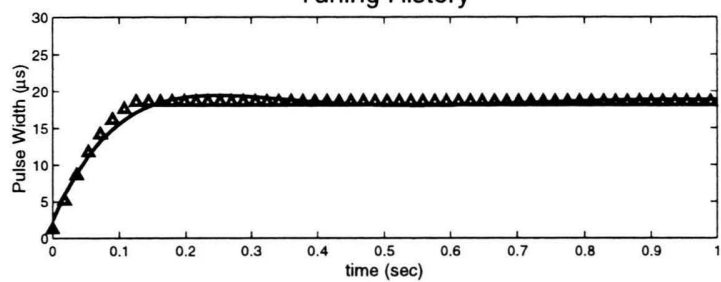

Fig. 7. Response of the SORC signal and the pulse width tuning history.

width of the $k^{\text {th }}$ RF pulse is denoted as $t_{\mathrm{w}}(k)$. The separation $\tau$ between consecutive RF pulses and the frequency of excitation are assumed to be fixed. Between the discrete-time indices $k$ and $k+1$, there are $N+2$ RF pulses of fixed width $t_{\mathrm{w}}(k)$ and pulse spacing $\tau$. The first $N$ SORC signals following the $k^{\text {th }}$ pulse are coherently added to produce a single, averaged, SORC response whose peak-to-peak amplitude is denoted as $V_{\text {avg }}(k)$. During the interval $2 \tau$ following the acquisition of the $N^{\text {th }}$ SORC signal, the feedback algorithm uses the measured signals $V_{\text {avg }}(k)$ and $V_{\text {avg }}(k-1)$ to determine the next pulse width $t_{\mathrm{w}}(k+1)$. Details of the tuning algorithm are described in [9].

The amplitude of the SORC signal and applied pulse width are shown in Fig. 7 for a control experiment using a fixed pulse separation of $1 \mathrm{~ms}$. The experiment was performed using a $70 \mathrm{~g}$ sample of sodium nitrite at room temperature. The frequency of the RF pulses was set near the $\nu_{-}$transition at 3.6 MHz. The amplitude of the SORC signal has been normalized with respect to the maximum SORC amplitude that can be obtained with a $1 \mathrm{~ms}$ pulse 
spacing. Each amplitude value represents the average of $N=16$ SORC waveforms. The initial pulse width $t_{\mathrm{w}}(0)$ was set to $1 \mu \mathrm{s}$. Within a dozen iterations the control algorithm automatically tuned the pulse width to maximize the amplitude of the SORC waveform.

\section{Conclusions}

A $1 \mathrm{~kW}$ pulsed spectrometer specifically designed for implementing feedback algorithms that automati-

[1] A. D. Hibbs, G. A. Barrall, P. V. Czipott, D. K. Lathrop, Y. K. Lee, E. E. Magnuson, R. Matthews, and S. A. Vierkotter. Landmine detection by nuclear quadrupole resonance. In Abinash C. Dubey, James F. Harvey, and Thomas Broach, editors, Detection and Remediation Technologies for Mines and Minelike Targets III, 1998, vol. 3392. p. 522-532.

[2] G. L. Petersen. Pulsed Nuclear Quadrupole Resonance Instrumentation and Study of the ${ }^{14} \mathrm{~N}$ Spectrum and Relaxation in Sodium Nitrite. $\mathrm{PhD}$ thesis, Brown University, June 1975.

[3] J. C. Harding, D. A. Wade, R. A. Marino, E. G. Sauer, and S. M. Klainer. J. Magn. Resonance, 36, 21 (1979).

[4] T. Hirschfeld and S. M. Klainer. J. Mol. Structure 58, 63 (1980). cally tune pulse parameters to maximize the SNR of NQR measurements was described. Automatic optimization off pulse parameters using feedback control is valuable in applications such as explosives detection, where the pulse parameters yielding the largest signal-to-noise cannot be determined beforehand.

\section{Acknowledgements}

This work was funded in part by the United States Army Construction Engineering Research Laboratories under contract DACA 88-95-K-0002.

[5] G. L. Petersen. Low frequency NQR matching network. The NQR Newsletter, 1(2): 20-23, February 1993. R. A. Marino, editor. Hunter College of C.U.N.Y.

[6] R. A. Marino and S. A. Klainer. J. Chem. Physics 67, 3388 (1977).

[7] S. S. Kim, J. R. P. Jayakody, and R. A. Marino. Z. Naturforsch. 47a, 415 (1992).

[8] A. J. Blauch, J. L. Schiano, and M. D. Ginsberg. J. Magn. Resonance 139, 139 (1999).

[9] J. L. Schiano, T. Routhier, A. J. Blauch, and M. D. Ginsberg. J. Magn. Resonance 140, 84 (1999).

[10] J. L. Schiano, A. J. Blauch, and M. D. Ginsberg. Z. Naturforsch. 55a, 67 (2000). 